\title{
Uso dos periódicos do Portal CAPES pelos Programas de Pós- graduação da Faculdade de Odontologia de Araraquara - UNESP - no período de 2000 a 2005
}

\author{
Use of the CAPES Digital Library of Scientific Journals in the \\ Graduate Studies Program at the School of Dentistry of \\ Araraquara - UNESP - in the period from 2000 to 2005
}

\author{
Beatriz Valadares CENDON' \\ Gustavo SIVIERI-ARAÚJO \\ Maria Helena Matsumoto Komasti LEVES ${ }^{3}$ \\ Nádia Ameno RIBEIRO ${ }^{4}$ \\ Lucília Vilarino MOREIRA ${ }^{5}$ \\ Marley Cristina Chiusoli MONTAGNOLI ${ }^{6}$ \\ Fábio Luiz Camargo Villela BERBERT ${ }^{7}$
}

\section{RESUMO}

Este artigo apresenta o resultado de uma pesquisa que teve por objetivo principal verificar se o Portal de Periódicos CAPES atende às necessidades de informação de usuários na área de odontologia. Como revisão de literatura, o trabalho traça a evolução, a consolidação e as tendências das bibliotecas digitais de periódicos e analisa as pesquisas existentes sobre o uso do Portal de Periódicos CAPES por usuários da área de saúde e ciências correlatas. O estudo identificou 91 títulos de periódicos de odontologia citados nas referências bibliográficas das teses e dissertações defendidas no período de 2000 a 2005, na Faculdade de Odontologia da UNESP de Araraquara, os

1 Doutora em Library and Information Science, University of Texas System, UTS, Estados Unidos. Docente da Escola de Ciência da Informação, Departamento de Organização e Tratamento da Informação, Universidade Federal de Minas Gerais, UFMG, Brasil. Av. Antônio Carlos, 6627, Caixa Postal 1606, Pampulha, 30161 -970, Belo Horizonte, MG, Brasil. Correspondência para:/Correspondence to: B.V. CENDON. E-mail: <cendon@eci.ufmg.br>.

2 Doutor em Endodontia, Universidade Estadual Paulista Júlio de Mesquita Filho, UNESP, Brasil. Docente da Faculdade de Ciências da Saúde, Departamento de Odontologia, Universidade de Brasília, UnB, Brasil. Campus Universitário Darcy Ribeiro, Asa Norte, 70910-900, Brasilia, DF - Brasil. E-mail: <gustavosivieiri@uol.com.br)>

3 Bibliotecária, Biblioteca da Faculdade de Odontologia, Universidade Estadual Paulista - Araraquara, SP, Brasil. E-mail: $<$ dirstbd@foar.unesp.br>

4 Especialista em Historia da Cultura e da Arte, Universidade Federal de Minas Gerais, UFMG, Brasil. Graduação em andamento em Biblioteconomia, Universidade Federal de Minas Gerais, UFMG, Brasil. E-mail: <nameno@eci.ufmg.br>.

5 Graduação em andamento em Biblioteconomia, Universidade Federal de Minas Gerais, UFMG, Brasil. Aluna de iniciação cientifica pela Escola de Ciência da Informação, Universidade Federal de Minas Gerais, UFMG, Brasil. Av. Antônio Carlos, 6627, sala 4016a, Pampulha, 30161-970, Belo Horizonte, MG, Brasil. E-mail: <luciliavilarino@hotmail.com>.

- Bibliotecária da Seção Técnica de Aquisição e Tratamento da Informação da Faculdade de Odontologia do Campus de Araraquara, SP, Brasil. E-mail: <marley@foar.unesp.br>

7 Doutor em Endodontia, Universidade de São Paulo, USP, Brasil. Docente da Faculdade de Odontologia de Araraquara, Universidade Estadual Paulista Júlio de Mesquita Filho, UNESP, Brasil. Rua Humaitá, 1680, Centro, 14801-903 - Araraquara, SP - Brasil. Email: <fberbert@foar.unesp.br>.

Recebido em 5/4/2007 e aceito para publicação em 17/2/2009. 
quais foram comparados com aqueles que constam do Portal de Periódicos CAPES. Nos resultados são relacionados, para a área de odontologia, os títulos de periódicos citados mas não pertencentes ao Portal, os títulos do Portal não citados nos trabalhos e a frequência de citação de cada título. Essa análise propiciou, também, levantar dados sobre os periódicos de odontologia mais utilizados pelos alunos de pós-graduação da mesma faculdade, para verificar a adequação da coleção de periódicos da sua Biblioteca às necessidades de informação de seus usuários.

Palavras-chave: Portal de Periódicos CAPES; periódicos eletrônicos; bibliotecas digitais; bibliotecas universitárias; estudo de usuários.

\begin{abstract}
This paper presents the results of a study that aimed at verifying if the contents of the CAPES Digital Library of Scientific Journals attend the information needs of users in the area of dentistry. In the literature review, the article traces the evolution, consolidation and trends of digital libraries of electronic journals and analyses the research about the use of the CAPES Digital Library by scholars in the health sciences and correlated areas. The study identified 91 journal titles cited in the bibliography references of the thesis and dissertations defended in the period from 2000 to 2005 at the School of Dentistry of Araraquara, Sao Paulo State University (UNESP) which were compared with those present in the CAPES Digital Library. The results show, for the area of dentistry, the periodicals which were cited but were not in the CAPES Digital Library, the periodicals which were in the CAPES Digital Library but were not cited and the number of times each title was cited. This analysis also gathered data about the journal titles which have the highest citation rates by the graduate students in order to verify the adequacy of the library journal collection to the information needs of the users.
\end{abstract}

Keywords: CAPES Digital Library of Scientific Journals; e-journals; digital libraries; university libraries; user study.

\section{INTRODUÇÃO}

Fruto de um consórcio de bibliotecas e gerenciado pela Coordenadoria de Aperfeiçoamento de Pessoal do Ensino Superior (CAPES), o Portal de Periódicos CAPES é a principal biblioteca digital de periódicos científicos no Brasil.

A CAPES assume a responsabilidade pela compra de grande parte das revistas científicas, de agentes e distribuidores, para as bibliotecas brasileiras participantes. Até 1999, a iniciativa sofreu com demoras no repasse das verbas por parte da CAPES para as instituições consorciadas e com outros percalços gerados por uma crise cambial. Porém, em meados de 2000, a massiva entrada dos periódicos científicos eletrônicos no mercado brasileiro e a criação de uma infra-estrutura tecnológica do órgão gerenciador permitiram a sua consolidação e ampliação (Amorim; Vergueiro, 2006). Na corrente data, o Portal oferece acesso a mais de 120 bases de dados de referências bibliográficas e resumos e ao texto completo de mais de 12 mil periódicos nacionais e internacionais, em todas as áreas do conhecimento. Esses periódicos pertencem a cerca de 60 editoras e distribuidores, das quais quase metade oferece publicações na área de saúde.

O acesso e uso do Portal de Periódicos CAPES é destinado ao corpo docente e discente, a pesquisadores e a funcionários de 191 instituições de ensino superior e de pesquisa em todo o país. A disponibilização desses recursos informacionais tem em vista atingir objetivos tais como: a atualização dos conhecimentos da comunidade científica e tecnológica; a dinamização do processo do ensino e da pesquisa no Brasil; o aumento da quantidade, da qualidade, da visibilidade e da competitividade internacional da produção científica brasileira; e a promoção da internacionalização da nossa comunidade acadêmica. O Portal é, ainda, instrumento de democratização e de redução das disparidades de acesso à informação entre os pesquisadores das várias regiões do país que passam a ter igual oportunidade de acesso, com maior rapidez 
e comodidade, a um maior número de títulos de publicações periódicas e bases de dados (Periódicos..., 2008).

A motivação para a pesquisa aqui apresentada foi investigar a adequação do conteúdo do Portal como suporte à produção dos trabalhos científicos oriundos de pesquisadores na área de saúde, os quais estão entre os maiores usuários da literatura científica (Damásio, 2004; Maia; Cendón, 2005; Martins, 2006; Monteiro, 2005). O presente estudo identificou, dentre os títulos de periódicos citados nas referências bibliográficas das teses e dissertações defendidas no período de 2000 a 2005 na Faculdade de Odontologia de Araraquara - Universidade Estadual Paulista (UNESP) -, a frequência com que cada um foi citado, quais não constam do Portal de Periódicos CAPES e do acervo da Biblioteca, quais constam do Portal e não são citados, e a quais editoras/distribuidoras pertencem os periódicos não citados.

\section{OS PERIÓdICOS CIENTÍFICOS E A GLOBALIZAÇÃO ELETRÔNICA}

desenvolvimento das novas tecnologias de informação e da comunicação em rede, a partir da segunda metade do século XX, trouxe novas soluções para a otimização da produção, do acesso e da disseminação da literatura científica. Entre os avanços mais recentes, está a disponibilização do texto completo dos periódicos científicos em forma digital, os quais passaram, nesse formato, a serem denominados periódicos eletrônicos.

Oadvento dos periódicos eletrônicos gerou uma revolução na área da informação, com transformações para todos os envolvidos na produção, na disseminação e na utilização de informações, entre eles autores, editores, bibliotecários e usuários. Para o usuário, o periódico no formato eletrônico proporcionou, entre vários outros benefícios, maior facilidade e eficiência na busca, na recuperação e na obtenção dos artigos. Outros benefícios são as possibilidades criadas pela utilização do hipertexto, tais como a não-linearidade, a acessibilidade ilimitada, o uso de multimídia e a interatividade, que possibilitam inovações na apresentação de resultados de pesquisas. São ainda exemplos de vantagens as possibilidades: de acesso compartilhado em rede (o que elimina a necessidade de replicação de assinaturas das versões impressas); da atualização em tempo real das publicações; do acesso a coleções retrospectivas em uma única busca de informação; de uma maior proteção do acervo; de eliminação de custos do armazenamento físico; de supressão da depreciação do material e de melhor uso de recursos pela seleção dos artigos para posterior impressão, na íntegra ou em partes (Cruz et al., 2003; Damásio, 2004; Davenport, 1998; Dias, 2002; Lancaster, 1995; Levacov, 1997; Martins, 2006; Rogers, 2001).

Na década de 1980, uma conjugação de fatores contribuiu para acirrar o interesse de editores e bibliotecas pela construção de coleções on-line de jornais científicos. Amorim e Vergueiro (2006) arrolam, com base nos trabalhos de Brakel (1995), Meyer (1997), Odlyzko (1997), Rogers e Hurt (1990), Schauder (1994), algumas das causas motivadoras desse fenômeno:

- ineficácia do modelo tradicional: as informações científicas chegam ao seu público-alvo através de outros meios antes de sua efetiva publicação... (Brakel, 1995);

- limites físicos: alguns artigos, que poderiam trazer informações novas e relevantes, acabam não sendo publicados, devido à falta de espaço nas edições impressas... (Schauder, 1994);

- alta especialização e baixa circulação: há uma tendência global que torna excessivo o número atual de revistas científicas (algo típico da explosão informacional, característico das sociedades atuais), onde cada título, com raríssimas exceções, tem um público extremamente limitado e elitista... (Rogers e Hurt, 1990);

- altos e crescentes custos: a própria especialização e dificuldades ligadas à editoração forçam as publicações a terem tiragens pequenas e, como consequência, um baixo número de assinantes, criando assim um círculo vicioso que contribui para a elevação de custos e a inflação dos preços observados, indicam, em seus estudos, Brakel (1995), Meyer (1997), Schauder (1994), e, sobretudo, Odlyzko (1997);

- falta de espaço para armazenamento nas bibliotecas: o problema é comum às grandes universidades brasileiras, representando um custo adicional aos orçamentos locais (Amorin; Vergueiro, 2006).

Nos anos 80, paralelamente à crise que se vislumbrava no mercado de publicações impressas em 
função de fatores tais como os acima mencionados, a superação das barreiras técnicas e do custo de hospedagem on-line, os desenvolvimentos de software e a disseminação do uso de computadores pessoais e de redes (Arms, 2000.) viabilizaram as iniciativas de criação de bibliotecas digitais de periódicos científicos.

As primeiras tentativas para digitalizar a informação científica e torná-la disponível via redes tiveram início em 1988, com o projeto Mercury Eletronic Library da Carnegie Mellon University, que desenvolveu e implementou o software para uma biblioteca digital de periódicos oferecida por meio da biblioteca universitária. Posteriormente, outros projetos de pesquisa e desenvolvimento de bibliotecas digitais centrados no texto completo de periódicos eletrônicos surgiram, incluindo CORE (1990-1995), TULIP (1991-1995), Red Sage (1992-1996), MUSE (1994-), Super Journal (1995-) e JSTOR (1995-). Entre os projetos já concluídos, o CORE, da Cornell University, que terminou em 1995, disponibilizou o texto completo de 20 periódicos da American Chemical Society. O projeto TULIP incluiu 43 periódicos da Elsevier e Pergamon. Participantes no projeto Red Sage tinham acesso a 71 periódicos de 19 editoras disponíveis aos usuários de janeiro de 1994 a dezembro de 1996.

Os resultados dessas primeiras experiências, que exploraram o potencial de fornecer periódicos eletrônicos on-line, foram utilizados por grandes editores de revistas científicas como Academic Press, John Wiley, Elsevier Science, Springer-Verlag, Blackwell Scientific e também por grandes sociedades acadêmicas, como o American Institute of Physics (Bishop et al., 2000; Peek, 1996), que, em torno de 1996, começaram a disponibilizar suas publicações on-line, oferecendo aos assinantes acesso ao texto completo. Mais tarde, outras iniciativas institucionais apareceram, como a Highwire Press (http://highwire.stanford.edu/), uma venture da Stanford University Libraries que, através de várias parcerias, disponibiliza on-line periódicos em ciência, tecnologia e medicina.

Cunha (1997) ao explorar as vantagens e desvantagens dos periódicos eletrônicos em suporte digital, concluiu que os e-journals haviam superado as principais barreiras técnicas para a sua consolidação, e poderiam ser uma alternativa ou, mesmo, substituir ou coexistir com os periódicos impressos. Confirmando a expectativa de Cunha, atualmente observa-se o florescimento do mercado das publicações eletrônicas.
Muitos periódicos abandonaram a forma impressa. Outros surgiram somente na forma digital, podendo ser disseminados por vários meios, por exemplo, através de listas de discussão, newsgroups, CD-ROMS ou por sites na web. O número de usuários vem crescendo a cada ano. Em pesquisa com estudantes da Ohio State University, Sally A. Rogers (2001) mostrou que o percentual de uso de periódicos eletrônicos havia crescido de 36,2\% em 1998 para 53,9\% em 2000. Ao mesmo tempo, o número de respondentes que reportaram o uso de periódicos impressos havia caído de $74,3 \%$ para $65,6 \%$ no mesmo período. Também o estudo de Lawrence (2001) atesta o sucesso das publicações eletrônicas, ao mostrar que, dentro da ciência da computação, publicações eletrônicas são mais citadas que as em papel, sendo a média de citações para artigos impressos 2,74 enquanto a de artigos on-line é de 7,03 (Marcondes; Sayão, 2002).

Mais recentemente, no mercado das publicações científicas eletrônicas, como reportam Amorim e Vergueiro (2006), com a repercussão do fenômeno da globalização, surge a tendência do controle do mercado das revistas científicas por oligopólios e da formação de grandes empresas transnacionais de informação, que, em geral, são resultado da incorporação de outras organizações e atuam em nível mundial:

No caso dos grandes agentes distribuidores atuantes na América Latina (Swets-Blackwell, Ebsco Information Services, Elsevier Science etc.), encontramos um mercado global caracterizado pela existência de um oligopólio ou monopólios onde exercem o poder duas ou mais empresas distribuidoras fornecendo diversos serviços de informação, entre eles o de periódicos científicos (Amorim; Vergueiro, 2006, p.38).

Segundo os mesmos autores, são detectadas no Brasil duas grandes empresas, a Wolters Kluwer, com sede na Holanda, e a Thompson Corporation, com sede nos Estados Unidos, que controlam "a quase totalidade do mercado local brasileiro de produtos informacionais para bibliotecas especializadas e universitárias" (Amorim, 2002, p.61).

Amorim e Vergueiro (2006) observam ainda que as pressões econômicas e metas comerciais dos agentes distribuidores e grandes editores interferem nas atividades de seleção e aquisição de periódicos científicos pelas 
bibliotecas. Uma prática desses agentes é o oferecimento de pacotes de periódicos (bundled EJournals). Exemplificando, no caso do pacote oferecido pela biblioteca digital ScienceDirect, da editora Elsevier, o custo da assinatura conjunta de um grande número de títulos de periódicos torna-se atraente. A Elsevier oferece opções de assinatura para bibliotecas que desejam uma coleção apenas em forma eletrônica; ou que apenas desejam complementar a coleção impressa com títulos eletrônicos por um valor bem menor do que a assinatura impressa. Em todos os casos, entretanto, não há flexibilidade ou torna-se antieconômico o cancelamento de títulos pouco ou raramente usados e que poderiam ser substituídos por periódicos de outras editoras. Algumas vezes os contratos permitem às instituições realizarem cancelamentos de títulos, porém exigem a sua substituição por outros periódicos da ScienceDirect de mesmo valor (Foster, 2002).

A formação de consórcios de bibliotecas ${ }^{8}$ é uma forma de aumentar o poder de negociação das bibliotecas junto aos grandes conglomerados do mercado de periódicos científicos. Entre as motivações para a criação do Portal de Periódicos CAPES, estava a possibilidade de facilitar, através do consórcio, a solução dos problemas enfrentados pelas instituições acadêmicas para manutenção da qualidade e a atualização de seus acervos bibliográficos.

\section{PESQUISAS SOBRE O USO DO PORTAL DE PERIÓDICOS CAPES}

A presente sessão discute as pesquisas que têm sido realizadas sobre o Portal e contextualiza o corrente trabalho. A seguir são mostrados, de forma sucinta, os resultados dos trabalhos com especial ênfase naqueles focalizados na área de saúde e ciências correlatas.

\section{Estudos de uso e satisfação de usuários do Portal de Periódicos CAPES}

Os estudos de uso e satisfação de usuários do Portal incluem pesquisas que têm por objetivo verificar o grau de aceitação e de satisfação dos usuários com a interface, funcionalidades oferecidas, desempenho do sistema, divulgação, adequação da seleção de títulos às suas necessidades e idioma dos títulos, entre outros itens (Cendón; Ribeiro, 2008). A maioria desses estudos, realizados em universidades, com amostras que incluíam usuários de todas as áreas do conhecimento, revela um alto percentual de uso do Portal, como exemplificado pelas pesquisas realizadas na Universidade Federal de Santa Catarina (UFSC), na Universidade Federal de Minas Gerais (UFMG), na Universidade Federal do Acre (UFAC) e na Universidade Federal do Mato Grosso (UFMT), a seguir descritas.

A UFSC foi estudada por Dutra e Lapolli (2004), que analisou o comportamento dos alunos e dos professores dos cursos de pós-graduação frente ao Programa de Apoio à Aquisição de Periódicos da CAPES. O autor apresenta os resultados de forma geral, sem se referir especificamente aos respondentes da área de saúde. Foram enviados dois mil questionários e obtidas 452 respostas, o que equivale a uma taxa de retorno de $22,6 \%$. O resultado da pesquisa, realizada em 2003, apresentou um cenário positivo com relação à aceitação e ao uso de novas tecnologias e de novos serviços. Os dados mostram que 90,8\% dos usuários acessavam o Portal CAPES, e desses, 74,8\% o faziam uma ou mais de uma vez por semana. Tal resultado mostrou, também, que uma parcela considerável dos usuários $(79,7 \%)$ não conhece todos os recursos disponíveis no Portal de Periódicos CAPES.

Maia e Cendón (2005) estudaram o uso do Portal por professores da UFMG em todas as áreas do conhecimento. Entre os 152 respondentes, 87,3\% já haviam utilizado o Portal CAPES enquanto $12,7 \%$ nunca o haviam acessado. 92,4\% dos respondentes estavam satisfeitos com a qualidade dos periódicos disponíveis no Portal em suas áreas de conhecimento, mas apenas $44,9 \%$ declararam-se satisfeitos em relação à sua quantidade. Apontaram, como dificuldades e barreiras para o uso, a lentidão do acesso ao sistema e as dificuldades de acesso fora da UFMG, principalmente.

Reis (2005), em sua dissertação de mestrado, analisou o acesso e o uso do Portal por 77 docentes, professores da Universidade Federal do Acre (UFAC), de várias áreas do conhecimento. 67,6\% dos respondentes acessavam o Portal, enquanto $22,1 \%$ nunca o haviam consultado. Os respondentes

8 Tais consórcios surgiram nos Estados Unidos em meados do século XVII, mas apenas a partir da década de 80 emergem no Brasil e América Latina (Amorim; Vergueiro, 2006). 
consideravam importante a quantidade de periódicos $(72,7 \%)$, a ausência de custo para o uso do Portal $(70,2 \%)$, a atualidade dos periódicos $(76,6 \%)$ e a facilidade de uso da rede (73,2\%). 54,5\% deles mencionaram que o Portal atende às suas necessidades. Consideravam fatores dificultadores para o uso do Portal a falta de habilidade dos usuários no manuseio dos recursos e os problemas para acesso.

A UFMT foi estudada por Pinheiro; Silva e Diniz (2006). Dos 38 docentes do período noturno do Campus Rondonópolis (que representavam 70\% do total), $55 \%$ responderam que usavam o Portal. $45 \%$ declararam que o desconheciam, apesar de muitos do Campus Rondonópolis indicarem que usavam outras fontes eletrônicas.

\section{Estudos de uso e satisfação de usuários na área de saúde e ciências correlatas}

Poucos trabalhos mostram resultados especificamente para a área de saúde. Os principais encontrados na literatura pesquisada foram os de Damásio (2004); Maia e Cendón (2005); Martins (2006) e Monteiro (2005), que apresentam alguns resultados comuns no que toca à reivindicação de aumento no número de periódicos e de necessidade de maior treinamento para os usuários. De um modo geral, mostram que os profissionais da área da saúde estão entre os maiores utilizadores do Portal.

No estudo de Maia e Cendón (2005), na UFMG, os dados demonstraram que 94,4\% dos respondentes da área de saúde utilizavam o Portal CAPES, dos quais $82,4 \%$ estavam satisfeitos com a qualidade dos periódicos da sua área disponíveis no Portal. Entretanto, apenas 33,3\% destes declararamse satisfeitos com a quantidade de periódicos disponíveis.

Damásio (2004) realizou pesquisa com 21 alunos do Curso de Mestrado em Ciências Farmacêuticas da Universidade Estadual de Maringá. O estudo mostra que $93 \%$ dos respondentes consideravam os periódicos e bases de dados do Portal ótimos para suas pesquisas e revisões bibliográficas. Corroborando os resultados de Maia e Cendón, a pesquisa mostra que a quantidade de periódicos disponibilizados deveria ser ainda maior e que os investimentos para aumento do número de periódicos devem continuar.

Monteiro (2005), que realizou seu estudo com pesquisadores, docentes, alunos de pós-graduação e bibliotecários da Universidade de Brasília (UnB) e da Universidade Federal de Goiás (UFG), teve por objetivo identificar o grau de satisfação dos usuários do Portal em relação à divulgação, à seleção de títulos de periódicos em sua área, à velocidade de acesso, ao idioma dos títulos de periódicos e à apresentação gráfica do site do Portal. Os programas pesquisados foram os de Medicina Tropical, Direito, Educação, Matemática e Engenharia Elétrica. Os usuários da área de Medicina Tropical estavam entre os maiores usuários do Portal e os que mais participaram das atividades de treinamento. Estavam também entre aqueles que mais acreditavam que $\circ$ Portal tem influenciado suas atividades de pesquisa e a sua produtividade. 56,1\% estavam muito satisfeitos com a capacidade do Portal de atender às suas necessidades informacionais.

O estudo de Martins (2006) analisou o uso do Portal por pesquisadores doutores da área biomédica da Universidade Federal do Rio de Janeiro. Constatouse que $94 \%$ dos pesquisadores tornaram-se usuários do Portal logo que ele se instalou, em 2000. Questionados sobre a satisfação com relação aos periódicos científicos disponíveis, os pesquisadores manifestaram-se pela inclusão de novos títulos e editoras, e pela ampliação da cobertura em diversas áreas do conhecimento. 34\% não estão completamente satisfeitos com a core list.

Deve-se ressaltar, entretanto, que a pesquisa de Martínez-Silveira e Oddone (2005), ao contrário dos resultados dos estudos acima relatados, revela pouca intimidade dos residentes médicos com os recursos informacionais em forma eletrônica, mostrando escassa utilização de Portais e de bases de dados médicas e o manejo deficiente de seus recursos. Embora essa pesquisa não seja especificamente sobre o Portal de Periódicos CAPES, esse resultado parece contrastar com aqueles acima reportados, que mostram os pesquisadores da área de saúde como grandes e, em sua maioria, satisfeitos utilizadores do Portal. 


\section{Estudos de impacto do Portal na pesquisa e produtividade / Adequação do Portal CAPES}

Outros trabalhos têm o propósito de estudar a relação entre a implantação e o uso do Portal e a evolução do volume e da qualidade da produção intelectual brasileira, bem como de avaliar programas de pós-graduação. Alguns deles verificam, também, a adequação, a representatividade e a abrangência dos periódicos do Portal para determinadas áreas do conhecimento (Cendón; Ribeiro, 2008).

Dentro da área biomédica, o estudo de Sakabe 9 (2006) pertence a essa categoria. Sakabe (2006) verifica se, por meio do Portal, um pesquisador brasileiro teria acesso ao texto completo das referências bibliográficas citadas em artigos de alto nível presentes na literatura internacional. $O$ pressuposto é que $O$ acesso às publicações citadas em um artigo é efetivamente necessário para a sua produção. O autor analisou 10 artigos da área de ciências biológicas publicados em revistas internacionais de alto nível e calculou a porcentagem de referências citadas que podem ser efetivamente lidas por pesquisadores brasileiros por meio do Portal. Concluiu que o Portal praticamente dobra a quantidade de artigos acessíveis, em relação aos que podem ser obtidos gratuitamente via internet. Dessa forma, a pesquisa mostra claramente que o Portal de Periódicos CAPES possui um importante papel no acesso a artigos acadêmicos em todo o território nacional, possibilitando, em conjunto com as revistas de acesso aberto, que os pesquisadores brasileiros tenham à disposição boa parte da literatura utilizada para redigir artigos de alto nível, ao menos no que tange à área de Biologia, nas condições apresentadas.

\section{Estudos bibliométricos e de citações dos periódicos do Portal CAPES}

Outra forma de analisar o uso dos periódicos disponibilizados por meio do Portal e sua adequação às necessidades dos seus usuários são os estudos bibliométricos dos trabalhos publicados pela comunidade de usuários do Portal. A bibliometria é um método quantitativo de investigação da ciência, sendo a análise de citações uma de suas ferramentas. Por meio do cômputo e da análise das citações dos periódicos na produção científica das instituições de ensino e pesquisa, pode-se caracterizar e mapear o uso de bibliotecas digitais, tais como o Portal CAPES.

A premissa básica da análise de citações é que os cientistas mencionam outros trabalhos em seus textos. Há uma variedade de motivos para citação, por parte de um pesquisador, de trabalhos de outros, entre os quais: dar crédito a trabalhos, métodos ou teorias que serviram de inspiração, ou foram utilizados pelo autor no desenvolvimento de seu próprio trabalho; prestar homenagem aos pioneiros; oferecer leitura básica; analisar trabalhos anteriores; sustentar declarações; identificar publicações originais nas quais uma idéia ou um conceito são discutidos; contestar trabalhos ou idéias de outros e expor a literatura que é indispensável para o trabalho do cientista (Carvalho, 1975; Noronha, 1998; Vanz; Caregnato, 2003; Weinstock, 1971). outra possibilidade é ordenar cronologicamente, mas assim for, deve-se manter uma ordem única no texto todo.

Os estudos de citações permitem análises aprofundadas do processo de comunicação científica. Por exemplo, pode-se determinar o impacto e a visibilidade de determinados autores dentro de uma comunidade científica; verificar quais "escolas" do pensamento, teorias e metodologias estão consolidadas em determinada área e identificar características das fontes de informação utilizadas, como o tipo de documento, o idioma e os periódicos mais citados (Vanz e Caregnato, 2003). O estudo das citações pode revelar, ainda, as realizações científicas de indivíduos, grupos, instituições ou países. Podem também mostrar a evolução de um campo específico (Moravcsik; Murugesan, 1975), permitir o estudo das características dos procedimentos de busca e recuperação da informação (Peritz, 1992) ou comportamentos dos cientistas, suas tendências de concentração em campos, em instituições, em países e no uso de determinados periódicos (Morel; Morel, 1977). Destaca-se, como particularmente importante no contexto do presente trabalho, que a análise de citações pode ser utilizada na avaliação do uso de publicações.

\footnotetext{
- SAKABE, N. J. Influência do Portal de Periódicos da CAPES na Pós-Graduação Brasileira: análise da cobertura do Portal de Periódicos baseada na disponibilidade de artigos em publicações de alto nível. 2006. Não publicado.
} 
Dentro dessa linha, Sampaio ${ }^{10}$ (2006) analisa as referências citadas pelas 793 dissertações e teses defendidas nos cinco programas de pós-graduação dos cursos de pós-graduação do Instituto de Psicologia da Universidade de São Paulo (IPUSP), no período de 2000 a 2005. O impacto do Portal foi detectado a partir do aumento das citações a periódicos durante os anos em questão. Comprovou-se que a maioria dos periódicos citados está disponível no Portal.

O corrente estudo, cujos resultados são apresentados a seguir, enquadra-se dentro desse tipo de pesquisa.

\section{O ESTUDO E SEUS RESULTADOS}

Do Portal de Periódicos CAPES, constavam, na data em que a corrente pesquisa foi realizada, 377 títulos de periódicos na área da saúde, dos quais 92 eram relacionados à Odontologia. No presente estudo, realizou-se uma comparação entre os títulos de periódicos citados nas referências bibliográficas das dissertações e teses defendidas na Faculdade de Odontologia de Araraquara (UNESP), no período de 2000 a 2005, com os periódicos eletrônicos da área de odontologia cadastrados no Portal de Periódicos CAPES, e com os títulos de periódicos que constavam do acervo da Biblioteca da unidade.

Conforme mostra a Tabela 1, foram defendidas na Faculdade de Odontologia de Araraquara, no período 2000-2005, um total de 184 dissertações de mestrado e 136 teses de doutorado ${ }^{11}$. Identificaram-se, primeiramente, os títulos de periódicos de Odontologia citados nas referências bibliográficas de cada dissertação e tese, criando-se uma lista que serviu como instrumento de comparação entre os títulos de periódicos citados nas dissertações e teses e aqueles cadastrados no Portal CAPES e, ainda, os presentes no acervo da Biblioteca da Faculdade de Odontologia de Araraquara - UNESP. Concomitantemente, o número de vezes que cada título de periódico foi citado nas teses e dissertações foi contabilizado, verificando-se que totalizaram 18.804 citações durante os 6 anos pesquisados. Tais informações são mostradas na Tabela 1.

Tabela 1. Teses e dissertações defendidas na Faculdade de Odontologia de Araraquara (UNESP) de 2000 a 2005.

\begin{tabular}{cccccc}
\hline Ano & $\begin{array}{c}\text { N. de } \\
\text { dissertações }\end{array}$ & $\begin{array}{c}\text { N. de } \\
\text { teses }\end{array}$ & $\begin{array}{c}\text { N. total } \\
\text { de citações }\end{array}$ & $\begin{array}{c}\text { N. médio } \\
\text { de citações }\end{array}$ & $\begin{array}{c}\text { N. de títulos do } \\
\text { Portal citados }\end{array}$ \\
\hline 2000 & 39 & 18 & 2.651 & 46,51 & 40 \\
2001 & 10 & 26 & 2.133 & 59,25 & 37 \\
2002 & 40 & 11 & 2.746 & 53,84 & 43 \\
2003 & 20 & 29 & 3.157 & 64,43 & 44 \\
2004 & 46 & 25 & 4.674 & 65,83 & 47 \\
2005 & 29 & 27 & 3.443 & 61,48 & 51 \\
\hline TOTAL & 184 & 136 & 18.804 & 58,76 & N/A \\
\hline
\end{tabular}

140
Embora com pequeno retrocesso no ano de 2002, observa-se ao longo dos anos uma tendência crescente no número médio de citações por trabalho. Os dados indicam, ainda, um aumento progressivo do número de títulos citados nas dissertações e teses as quais constam no Portal de Periódicos CAPES, no período de 2000 a 2005, excetuando-se 2001, período em que ocorreu uma pequena queda de $3 \%$, se comparado ao ano anterior.
Constatou-se, também, que, no período de 2000 a 2005, os pós-graduandos da Faculdade de Odontologia de Araraquara (UNESP) fizeram frequentes referências a alguns títulos que não constam no Portal de Periódicos CAPES. Dos 92 títulos cadastrados, referentes à área de Odontologia, apenas 62 foram citados, e 30 não o foram. A Tabela 2 abaixo relaciona esses títulos e as editoras a que pertencem.

10 SAMPAIO, M. I. C. Impacto do Portal de Periódicos da CAPES na Produção Científica: Dissertações e Teses do IPUSP. 2006. Não publicado.

1 Não foram incluídas na análise as teses de livre docência, uma vez que a partir de 2002 elas deixaram de ser depositadas na Biblioteca. 
Tabela 2. Títulos da área de odontologia que, apesar de constarem do Portal, não foram citados.

\begin{tabular}{|c|c|}
\hline Título do periódico & Distribuidor/Editora \\
\hline Avances en Odontoestomalogía & SciELO / Ediciones Avances \\
\hline BMC Oral Health & BioMed Central \\
\hline $\begin{array}{l}\text { BoneKEy-Osteovision : A Knowlodge Environment on } \\
\text { Bone, Mineral, Cartilage and Teeth for Clinicians and } \\
\text { Researchers }\end{array}$ & International Bone \& Mineral Society (IBMS) \\
\hline Clinical Orthodontics and Research & Blackwell \\
\hline Critical Reviews in Oral Biology and Medicine & $\begin{array}{l}\text { American Association for Dental Research } \\
\text { (AADR);International Association for Dental Research } \\
\text { (IADR) }\end{array}$ \\
\hline Dysphagia & Springer \\
\hline EMC Dentisterie & Science Direct / Elsevier \\
\hline EMC Odontologie & Science Direct \\
\hline EMC Stomatologie & Science Direct \\
\hline European Journal of Cancer. Part B, Oral Oncology & $\begin{array}{l}\text { Science Direct / European Association of Oral } \\
\text { Medicine; International Academy of Oral } \\
\text { Oncology; International Association of Oral Pathologists }\end{array}$ \\
\hline European Journal of Dental Education & $\begin{array}{l}\text { Blackwell / Association for dental Education in Europe } \\
\text { (ADEE) }\end{array}$ \\
\hline Evidence-Based Dentistry & OVID / Nature \\
\hline Implantodontie & Science Direct / Elsevier \\
\hline International Journal of Paediatric Dentistry - IAPD & $\begin{array}{l}\text { Blackwell / British Society of Paediatric } \\
\text { Dentistry; International Association of Paediatric Dentistry } \\
\text { (IAPD) }\end{array}$ \\
\hline Journal of Contemporary Dental Practice & P\&G Professional Oral Health \\
\hline Journal of Craniofacial Surgery & $\begin{array}{l}\text { OVID / Korean Society of Craniofacial Surgery; European } \\
\text { Society of Craniofacial Surgery; Japanese Society of } \\
\text { Craniofacial Surgery;American Society of Craniofacial } \\
\text { SurgeonsInternational Society of Craniofacial } \\
\text { Surgery;American Association of Pediatric Plastic } \\
\text { Surgeons;American Academy of Pediatrics. Section of } \\
\text { Pediatric Plastic Surgery }\end{array}$ \\
\hline Journal of Evidence-Based Dental Practice & Science Direct / Mosby \\
\hline Journal of Orthodontics & Maney Publishing / British Orthodontic Society \\
\hline Mund-, Kiefer- und Gesichtschirurgie & Springer \\
\hline New York State Dental Journal : NYSDJ & New York State Dental Association (NYSDA) \\
\hline Odontology & Springer \\
\hline Oral Oncology Extra & $\begin{array}{l}\text { Science Direct / European Association of Oral } \\
\text { Medicine; International Academy of Oral Oncology; } \\
\text { International Association of Oral Pathologists }\end{array}$ \\
\hline Oral Oncology. Supplement & Science Direct \\
\hline $\begin{array}{l}\text { Orthodontics and Craniofacial Research : Genes, Growth } \\
\text { and Development }\end{array}$ & Blackwell \\
\hline
\end{tabular}


continuação

Título do Periódico

Pesquisa Brasileira em Odontopediatria e Clínica

Integrada

Progress in Orthodontics : Mediterraneam Journal of Orthodontics

Revista Española de Cirugía Oral y Maxilofacial

Revista Estomatológica Herediana

Sleep and Breathing

\section{Distribuidor/Editora}

Associação de Apoio à Pesquisa em Saúde Bucal (APESB)

Società Italiana di Ortodonzia

SciELO / Sociedad Española de Cirugía Oral y

Maxilofacial

SciELO / Universidad Peruana Cayetano Heredia.

Facultad de Estomatología

Springer / Academy of Dental Sleep Medicine (ADSM);

European Dental Sleep Medicine Academy (EdeSA);

British Society of Dental Sleep Medicine (BSDSM);

Deutsche Gesellschaft Schlaftherapeutisch

Tätiger Zahnmediziner (DGSZ)

Journal of Biomechanics

Science Direct / European Society of Biomechanics

American Society of Biomechanics (ASB)

International Society of Biomechanics (ISB)

Japanese Society for Clinical Biomechanics (JSCB)

Australian and New Zealand Society of Biomechanics

Dos 91 títulos citados, 29 não estavam disponíveis no Portal, como mostra a Tabela 3. Deles emanaram 35,66\% das citações. Considerando a frequência com que foram citados, alguns poderiam ser potenciais candidatos a incorporação futura pelo Portal, caso se verifique que são também importantes para usuários de outras instituições.

Tabela 3. Títulos que, apesar de não constarem do Portal de Periódicos CAPES, foram citados nas teses e dissertações defendidas na Faculdade de Odontologia de Araraquara (UNESP) de 2000 a 2005.

\section{Título do Periódico}

N. citações

$\%$ do $n$. total de citações (18.804)

Journal of Periodontology

Journal of the American Dental Association

Operative Dentistry

American Journal of Dentistry

Quintessence International

American Journal of Orthodontics

British Dental Journal

Acta Odontologica Scandinavica

International Journal of Prosthodontics

Dental Clinics of North America

Revista Gaúcha de Odontologia: RGO

Revista Brasileira de Odontologia

International Dental Journal

Scandinavian Journal of Dental Research

International Journal of Periodontics Restorative

Dentistry
1.370

716

624

581

497

483

295

259

246

152

152

146

137

137

115
7,29

3,81

3,32

3,09

2,64

2,57

1,57

1,38

1,31

0,81

0,81

0,78

0,73

0,73

0,61

TransInformação, Campinas, 21 (2): p. 133-149, maio/ago., 2009 
International Journal of Oral Maxillofacial Implants

$\begin{array}{rr}113 & 0,60 \\ 112 & 0,60 \\ 106 & 0,56 \\ 92 & 0,49 \\ 86 & 0,46 \\ 53 & 0,28 \\ 51 & 0,27 \\ 48 & 0,26 \\ 45 & 0,24 \\ 28 & 0,15 \\ 20 & 0,11 \\ 20 & 0,11\end{array}$

Journal of Biomedical Materials Research

Pediatric Dentistry

0,56

Lasers in Surgery and Medicine

0,49

Journal of Adhesive Dentistry

Journal of Clinical Laser Medicine \& Surgery

0,28

British Journal of Orthodontics

0,27

Journal of Clinical Orthodontics

0,26

Journal of Dentistry for Children

0,24

Ortodontia

0,15

Applied and Environmental Microbiology

Dentistry

Journal of Public Health Dentistry

A análise dos dados possibilitou também a verificação de quais títulos são os mais citados e, portanto, potencialmente recomendados para inclusão no Portal e para aquisição pela Biblioteca. A Tabela 4 mostra a totalidade dos títulos citados, a sua presença na biblioteca e no Portal, o número de vezes em que foram citados, o percentual do total e o percentual acumulado de citações. Nota-se que praticamente $80 \%$ (79,84\%) das citações pertencem a 22 títulos, dos quais o Portal possuía apenas 14 em 2005, enquanto, com 56 dos 92 títulos, cobrem-se praticamente $98 \%$ das citações.

Tabela 4. Títulos citados em relação à disponibilidade no Portal e na Biblioteca e o número e percentagem de citações.

\begin{tabular}{c|l|c|c|c|c|c}
\hline N. & \multicolumn{1}{|c|}{ Título do Periódico } & Biblioteca & Portal & TOTAL & $\%$ & $\%$ Acum. \\
\hline 01 & Journal of Endodontics & $\mathrm{x}$ & $\mathrm{x}$ & 1.831 & 9,74 & 9,74 \\
\hline 02 & Journal of Dental Research & $\mathrm{x}$ & $\mathrm{x}$ & 1.484 & 7,89 & 17,63 \\
\hline 03 & Journal of Prosthetic Dentistry & $\mathrm{x}$ & $\mathrm{x}$ & 1.451 & 7,72 & 25,35 \\
\hline 04 & Journal of Periodontology & $\mathrm{x}$ & & 1.370 & 7,29 & 32,63 \\
\hline 05 & Journal of Clinical Periodontology & $\mathrm{x}$ & $\mathrm{x}$ & 851 & 4,53 & 37,16 \\
\hline 06 & International Endodontic Journal & $\mathrm{x}$ & $\mathrm{x}$ & 759 & 4,04 & 41,19 \\
\hline 07 & Journal of the American Dental Association & & & 716 & 3,81 & 45,00 \\
\hline 08 & Dental Materials & $\mathrm{x}$ & $\mathrm{x}$ & 682 & 3,63 & 48,63 \\
\hline 09 & Operative Dentistry & & & 624 & 3,32 & 51,95 \\
\hline 10 & Journal of Dentistry & $\mathrm{x}$ & $\mathrm{x}$ & 608 & 3,23 & 55,18 \\
\hline 11 & American Journal of Dentistry & $\mathrm{x}$ & $\mathrm{x}$ & 524 & 2,79 & 61,06 \\
\hline 12 & American Journal of Orthodontics and & $\mathrm{x}$ & $\mathrm{x}$ & 519 & 2,76 & 63,82 \\
\hline 13 & Dentofacial Orthopedics & & & 3,09 & 58,27 \\
\hline
\end{tabular}




\section{continuação}

\begin{tabular}{|c|c|c|c|c|c|c|}
\hline N. & Título do Periódico & Biblioteca & Portal & TOTAL & $\%$ & $\%$ Acum. \\
\hline 14 & Quintessence International & & & 497 & 2,64 & 66,46 \\
\hline 15 & American Journal of Orthodontics & & & 483 & 2,57 & 69,03 \\
\hline 16 & Journal of Oral Rehabilitation & & $x$ & 366 & 1,95 & 70,97 \\
\hline 17 & Journal of Periodontal Research & $x$ & $x$ & 312 & 1,66 & 72,63 \\
\hline 18 & British Dental Journal & & & 295 & 1,57 & 74,20 \\
\hline 19 & Angle Orthodontist & $x$ & $x$ & 291 & 1,55 & 75,75 \\
\hline 20 & Archives of Oral Biology & $x$ & $x$ & 265 & 1,41 & 77,16 \\
\hline 21 & Acta Odontologica Scandinavica & & & 259 & 1,38 & 78,54 \\
\hline 22 & International Journal of Prosthodontics & & & 246 & 1,31 & 79,84 \\
\hline 23 & APCD Revista & & $x$ & 186 & 0,99 & 80,83 \\
\hline 24 & Clinical Oral Implants Research & $x$ & $x$ & 152 & 0,81 & 81,64 \\
\hline 25 & Dental Clinics of North America & & & 152 & 0,81 & 82,45 \\
\hline 26 & Revista Gaúcha de Odontologia: RGO & & & 152 & 0,81 & 83,26 \\
\hline 27 & Revista Brasileira de Odontologia & & & 146 & 0,78 & 84,04 \\
\hline 28 & $\begin{array}{l}\text { Oral Surgery, Oral Medicine, Oral Pathology, } \\
\text { Oral Radiology and Endodontics }\end{array}$ & & $x$ & 143 & 0,76 & 84,80 \\
\hline 29 & Revista de Odontologia da UNESP & & $x$ & 140 & 0,74 & 85,54 \\
\hline 30 & International Dental Journal & & & 137 & 0,73 & 86,27 \\
\hline 31 & Scandinavian Journal of Dental Research & & & 137 & 0,73 & 87,00 \\
\hline 32 & Caries Research & $x$ & $x$ & 136 & 0,72 & 87,72 \\
\hline 33 & $\begin{array}{l}\text { International Journal of Periodontics and } \\
\text { Restorative Dentistry }\end{array}$ & & & 115 & 0,61 & 88,33 \\
\hline 34 & $\begin{array}{l}\text { International Journal of Oral and Maxillofacial } \\
\text { Implants }\end{array}$ & & & 113 & 0,60 & 88,93 \\
\hline 35 & Journal of Biomedical Materials Research & & & 112 & 0,60 & 89,53 \\
\hline 36 & Australian Dental Journal & & $x$ & 108 & 0,57 & 90,10 \\
\hline 37 & European Journal of Orthodontics & & $\mathrm{x}$ & 108 & 0,57 & 90,68 \\
\hline 38 & Pediatric Dentistry & & & 106 & 0,56 & 91,24 \\
\hline 39 & European Journal of Oral Sciences & & $x$ & 105 & 0,56 & 91,80 \\
\hline 40 & Pesquisa Odontológica Brasileira & & $x$ & 102 & 0,54 & 92,34 \\
\hline 41 & Lasers in Surgery and Medicine & & & 92 & 0,49 & 92,83 \\
\hline 42 & $\begin{array}{l}\text { Revista de Odontologia da Universidade de São } \\
\text { Paulo }\end{array}$ & & $x$ & 89 & 0,47 & 93,30 \\
\hline 43 & Oral Microbiology and Immunology & & $x$ & 87 & 0,46 & 93,77 \\
\hline 44 & Journal of Adhesive Dentistry & & & 86 & 0,46 & 94,22 \\
\hline 45 & Journal of Oral and Maxillofacial Surgery & & $x$ & 84 & 0,45 & 94,67 \\
\hline 46 & Community Dentistry and Oral Epidemiology & & $\mathrm{x}$ & 81 & 0,43 & 95,10 \\
\hline 47 & Periodontology 2000 & & $x$ & 76 & 0,40 & 95,51 \\
\hline 48 & Brazilian Dental Journal & & $x$ & 64 & 0,34 & 95,85 \\
\hline 49 & Revista da Faculdade de Odontologia de Bauru & & $x$ & 64 & 0,34 & 96,19 \\
\hline 50 & $\begin{array}{l}\text { Journal of the Canadian Dental Association: } \\
\text { JCDA }\end{array}$ & & $x$ & 57 & 0,30 & 96,49 \\
\hline
\end{tabular}




\begin{tabular}{|c|c|c|c|c|c|c|}
\hline N. & Título do Periódico & Biblioteca & Portal & TOTAL & $\%$ & $\%$ Acum. \\
\hline 51 & Journal of Clinical Laser Medicine \& Surgery & & & 53 & 0,28 & 96,77 \\
\hline 52 & British Journal of Orthodontics & & & 51 & 0,27 & 97,04 \\
\hline 53 & Journal of Clinical Orthodontics & & & 48 & 0,26 & 97,30 \\
\hline 54 & Journal of Dentistry for Children & & & 45 & 0,24 & 97,54 \\
\hline 55 & Implant Dentistry & & $x$ & 44 & 0,23 & 97,77 \\
\hline 56 & Brazilian Oral Research & & $\mathrm{x}$ & 38 & 0,20 & 97,97 \\
\hline 57 & Advances in Dental Research & & $x$ & 30 & 0,16 & 98,13 \\
\hline 58 & Ortodontia & & & 28 & 0,15 & 98,28 \\
\hline 59 & $\begin{array}{l}\text { Revista Dental Press de Ortodontia e Ortopedia } \\
\text { Facial }\end{array}$ & & $\mathrm{x}$ & 26 & 0,14 & 98,42 \\
\hline 60 & Cleft Palate Journal & & & 23 & 0,12 & 98,54 \\
\hline 61 & $\begin{array}{l}\text { International Journal of Oral and Maxillofacial } \\
\text { Surgery }\end{array}$ & & $\mathrm{x}$ & 23 & 0,12 & 98,67 \\
\hline 62 & Applied and Environmental Microbiology & & & 20 & 0,11 & 98,77 \\
\hline 63 & $\begin{array}{l}\text { European Journal of Prosthodontics and } \\
\text { Restorative Dentistry }\end{array}$ & & & 20 & 0,11 & 98,88 \\
\hline 64 & Journal of Public Health Dentistry & & & 20 & 0,11 & 98,98 \\
\hline 65 & $\begin{array}{l}\text { International Journal of Adult Orthodontics and } \\
\text { Orthognathic Surgery }\end{array}$ & & $\mathrm{x}$ & 19 & 0,10 & 99,09 \\
\hline 66 & Revista Odontológica do Brasil Central: & & $\mathrm{x}$ & 17 & 0,09 & 99,18 \\
\hline 67 & $\begin{array}{l}\text { ROBRAC } \\
\text { Clinical Oral Investigations }\end{array}$ & & $\mathrm{x}$ & 16 & 0,09 & 99,26 \\
\hline 68 & Journal of Oral Pathology and Medicine & & $\mathrm{x}$ & 14 & 0,07 & 99,34 \\
\hline 69 & Seminars in Orthodontics & & $x$ & 14 & 0,07 & 99,41 \\
\hline 70 & Journal of Oral Science & & $x$ & 11 & 0,06 & 99,47 \\
\hline 71 & Dental Traumatology & $x$ & $\mathrm{x}$ & 10 & 0,05 & 99,52 \\
\hline 72 & Oral Diseases & & $x$ & 10 & 0,05 & 99,57 \\
\hline 73 & $\begin{array}{l}\text { Ciência Odontológica Brasileira = Brazilian } \\
\text { Dental Science }\end{array}$ & & $x$ & 9 & 0,05 & 99,62 \\
\hline 74 & Journal of Applied Oral Science: JAOS & & $\mathrm{x}$ & 9 & 0,05 & 99,67 \\
\hline 75 & British Journal of Oral and Maxillofacial Surgery & & $\mathrm{x}$ & 8 & 0,04 & 99,71 \\
\hline 76 & Journal of Dental Education: JDE & & $x$ & 7 & 0,04 & 99,75 \\
\hline 77 & Oral Oncology & & $\mathrm{x}$ & 7 & 0,04 & 99,79 \\
\hline 78 & Acta Odontológica Venezolana & & $x$ & 6 & 0,03 & 99,82 \\
\hline 79 & Pós-Graduação em Revista: PGR & & $\mathrm{x}$ & 5 & 0,03 & 99,85 \\
\hline 80 & Journal of Cranio-Maxillofacial Surgery & & $\mathrm{x}$ & 4 & 0,02 & 99,87 \\
\hline 81 & Revista de Odontologia da UNICID & & $x$ & 4 & 0,02 & 99,89 \\
\hline 82 & Journal of Dental Hygiene & & $\mathrm{x}$ & 3 & 0,02 & 99,90 \\
\hline 83 & $\begin{array}{l}\text { Journal of Materials Science: Materials in } \\
\text { Medicine }\end{array}$ & & $\mathrm{x}$ & 3 & 0,02 & 99,92 \\
\hline 84 & $\begin{array}{l}\text { Journal of Orofacial Orthopaedics }= \\
\text { Fortschritte der Kieferorthopadie }\end{array}$ & & $\mathrm{x}$ & 3 & 0,02 & 99,94 \\
\hline
\end{tabular}


continuação

\begin{tabular}{c|l|c|c|c|c|c}
\hline N. & Título do Periódico & Biblioteca & Portal & TOTAL & $\%$ & $\%$ Acum. \\
\hline 85 & Revista Cubana de Estomatologia & & $x$ & 3 & 0,02 & 99,95 \\
\hline 86 & Dental Assistant & & $x$ & 2 & 0,01 & 99,96 \\
\hline 87 & Journal - District of Columbia Dental Society & & & 2 & 0,01 & 99,97 \\
\hline 88 & Medicina Oral, Patología Oral y Cirugía Bucal & & $x$ & 2 & 0,01 & 99,98 \\
\hline 89 & Journal of Biomaterials Applications & & $x$ & 1 & 0,01 & 99,99 \\
\hline 90 & Oral Radiology & & $x$ & 1 & 0,01 & 99,99 \\
\hline 91 & RCOE & & $x$ & 1 & 0,01 & 100,00 \\
\hline & TOTAL & N/A & N/A & 18.804 & 100,00 & N/A \\
\hline
\end{tabular}

A Tabela 5 apresenta os títulos citados nas referências das dissertações de mestrado e teses de doutorado que constavam do acervo da Biblioteca da Faculdade de Odontologia de Araraquara (UNESP), no período de 2000 a 2005. Embora estivessem disponíveis na Biblioteca poucos títulos, 16 ao todo, eles respondem por $59,8 \%$ do total de citações. Deles, somente o Journal of Periodontology existia apenas na Biblioteca, enquanto os outros 15 estavam também disponíveis no Portal.

Tabela 5. Títulos que constam no acervo da Biblioteca (2000-2005) e que foram citados nas teses e dissertações defendidas na Faculdade de Odontologia de Araraquara (UNESP).

\section{Título do Periódico}

Journal of Endodontics

Journal of Dental Research

Journal of Prosthetic Dentistry

Journal of Periodontology

Journal of Clinical Periodontology

International Endodontic Journal

Dental Materials

Journal of Dentistry

American Journal of Orthodontics and

Dentofacial Orthopedics

Oral Surgery, Oral Medicine, Oral Pathology

Journal of Periodontal Research

Angle Orthodontist

Archives of Oral Biology

Clinical Oral Implants Research

Caries Research

Dental Traumatology
N. citações

1.831

1.484

1.451

1.370

851

759

682

608

524

519

312

291

265

152

136

10

$\%$ do n. total de citações (18.804)

TOTAL

11.245

9,74

7,89

7,72

7,29

4,53

4,04

3,63

3,23

2,79

2,76

1,66

1,55

1,41

0,81

0,72

0,05
$\mathrm{Na}$ Tabela 4, nota-se que os 6 títulos de periódicos mais citados nas referências das Dissertações e Teses defendidas no período de 2000 a 2005, a saber,
Journal of Endodontics; Journal of Dental Research; Journal of Prosthetic Dentistry; Journal of Periodontology; Journal of Clinical Periodontology; e International 
Endodontic Journal, que somam 7.746 citações $(41,21 \%$ do total), constam do acervo da Biblioteca da unidade.

Verificou-se que, entre 2005 - data em que os dados foram levantados - e 2008, 23 novos títulos foram adicionados ao Portal, na área de odontologia. Desses, entretanto, apenas seis foram citados nos trabalhos analisados. As inclusões foram: a continuação do American Journal of Orthodontics, agora intitulado American Journal of Orthodontics and Dentofacial Orthopedics; o International Dental Journal; o Journal of Periodontology; o Operative Dentistry; o Journal of the American Dental Association e a Revista Gaúcha de Odontologia: RGO.

\section{CONSIDERAÇÕES FINAIS}

Como visto, no período de 2000 a 2005 foram defendidas, na Faculdade de Odontologia de Araraquara, 184 dissertações de mestrado e 136 teses de doutorado. Comparando as referências bibliográficas dessas teses e dissertações com os periódicos que constavam do Portal de Periódicos CAPES em julho de 2006, verifica-se que o número de periódicos do Portal citados nesses trabalhos apresenta uma tendência de aumento gradativo ao longo dos anos, avançando de 40 para 51 títulos entre 2000 e 2005 . Percebe-se, também, uma tendência crescente no número médio de citações por trabalho, que aumentou de 46,5 para 61,5 no período estudado. Esses dados podem ser indicativos de um incremento progressivo da expressividade do Portal no atendimento às demandas do grupo de usuários em foco. $\bigcirc$ estudo parece indicar, ainda, que a divulgação do Portal CAPES, assim como o treinamento ${ }^{12}$ para a sua utilização, em meio ao universo acadêmico e científico, contribuiu para elevar a presença do Portal CAPES na comunidade de usuários da biblioteca. Indica ainda que, apesar das dificuldades financeiras, a Biblioteca mantém as assinaturas dos títulos principais para as pesquisas em desenvolvimento na Unidade de Ensino.
Dos resultados encontrados, merecem destaque a identificação, para a Faculdade de Odontologia de Araraquara, no período de 2000 a 2005, dos 30 títulos disponibilizados no Portal, mas que não foram citados (Tabela 2), dos 29 títulos de periódicos citados e que não constam do Portal de Periódicos CAPES (Tabela 3), das editoras às quais esses periódicos pertencem e da lista completa de periódicos citados com a frequência de citação (Tabela 4). $\bigcirc$ estudo mostra, também, que dos 23 títulos da área de odontologia adicionados ao Portal após 2005, apenas 6 encontram-se entre os citados nos trabalhos analisados.

Embora importantes, os dados relatados, por si só, são insuficientes para serem utilizados na avaliação da pertinência dos títulos contidos no Portal CAPES para a área de odontologia, por não emanarem de uma amostra representativa de todos os usuários da área. Ademais, muitas poderiam ser as razões que poderiam justificar esses dados. Por exemplo, é possível que os 30 periódicos não citados (Tabela 2) cubram áreas que não eram, no momento da pesquisa, objeto de interesse dos pesquisadores e docentes daquela faculdade específica. Por outro lado, alguns dos títulos da Tabela 4 podem ter sido citados em virtude da maior facilidade de acesso a eles, por estarem disponibilizados no Portal. Nesse caso, as suas citações não representariam, necessariamente, a preferência dos usuários por aqueles títulos em detrimento de outros. No entanto são desconhecidos os motivos que explicariam o uso ou não-uso dos periódicos e que são importantes para fundamentar decisões sobre a formação de coleções.

Assim sendo, uma recomendação advinda desse estudo é que pesquisas semelhantes sejam realizadas em outras instituições com programas de pósgraduação em odontologia, a fim de verificar o padrão de uso dos periódicos num universo mais abrangente. Além disso, para que se possa complementar, compreender e melhor explicar os padrões identificados, recomendam-se, particularmente, estudos qualitativos com os usuários do Portal.

12 A biblioteca fornece treinamento regulares para o uso do Portal Capes focalizando principalmente os alunos iniciantes da pósgraduação. Os cursos são extensivos aos demais usuários (docentes, alunos da graduação, residentes, estagiários, alunos da especialização). Oferece também, se necessário, treinamento individual. 


\section{REFERÊNCIAS}

AMORIM, A. M. A globalização do mercado de periódicos científicos eletrônicos e os consórcios de bibliotecas universitárias brasileiras: desafios à democratização do conhecimento científico. 2002. 147 f. Dissertação (Mestrado em Ciências da Comunicação) - Escola de Comunicação e Artes, Universidade de São Paulo. 2002.

AMORIM, A. M.; VERGUEIRO, W. Consórcios de bibliotecas no Brasil: um desafio à democratização do conhecimento. Perspectivas em Ciência da Informação, v. 1 1, n. 1, p.32-47, 2006.

ARMS, W. Y. Digital Libraries. Cambridge : The MIT Press, 2000.

BISHOP, Ann Peterson et al. Digital Libraries: situating use in changing information infrastructure. Journal of the American Society for Information Science, v. 51 , n. 4, p. 394-413, 2000.

BRAKEL, P. A. V. Electronic journal: publishing via Internet's WWW. Electronic Library, v. 13, n.4, p. 389-395, 1995.

CARVALHO, Maria Martha de. Análises Bibliométricas da Literatura de Química no Brasil. Ciência da Informação, v. 4, n. 2, p. 119-141, 1975.

CENDÓN, Beatriz Valadares; RIBEIRO, Nádia Ameno . Análise da literatura acadêmica sobre o Portal de Periódicos CAPES. Informação \& Sociedade: Estudos, v. 18, p. 157-178, 2008.Disponível em: <http://www.ies.ufpb.br/ojs2/index.php/ies/article/ view/1784/2128>. Acesso em: 14 nov. 2008.

CUNHA, Léo. Publicações científicas por meio eletrônico: critérios, cuidados, vantagens e desvantagens. Perspectivas em $\mathrm{Ci}$ ência da Informação, v. 2, n. 1, p. 77-92, 1997.

CRUZ, A. A. A. C. et al. Impacto dos periódicos eletrônicos em bibliotecas universitárias. Ciência da Informação, v. 32, n. 2, p. 47-53, 2003

DAMÁSIO, E. A visão dos pós-graduandos em Ciências Farmacêutica na utilização do Potal de Periódicos da CAPES e recursos do diretório de bases online da biblioteca central-UEM: a importância da capacitação realizada por bibliotecários. In: SEMINÁRIO NACIONAL DE BIBLIOTECAS UNIVERSITÁRIAS, 13., 2004, Natal. Anais eletrônicos... Natal : UFRN, 2004.

DAVENPORT, T. Ecologia da informação: por que só a tecnologia não basta para o sucesso na era da informação. 5. ed. São Paulo: Futura, 1998.

DIAS, Guilherme Ataíde. Periódicos eletrônicos: considerações relativas à aceitação deste recurso pelos usuários. Ciência da Informação, v. 31, n. 3, p. 18-25, 2002.

DUTRA, S. K. W.; LAPOLLI, E. M. Portal de Periódicos da CAPES: análise do uso na Universidade Federal de Santa Catarina. In: SEMINÁRIO NACIONAL DE BIBLIOTECAS UNIVERSITÁRIAS, 13., 2004, Natal. Anais eletrônicos... Natal : UFRN, 2004.

FOSTER, A. L. Second thoughts on 'bundled' e-Journals. The Chronicle of Higher Education, Sept. 20, 2002. Disponível em: <http://chronicle.com/free/v49/i04/04a03101.htm> Acesso em: 24 ago 2007.

LANCASTER, F. W. The evolution of electronic publishing. Library Trends, v. 43, n. 4, p. 518-527, 1995.

LAWRENCE, S. Free online availability substantially increases a paper's impact. Nature. Disponível em: < http://www.nature.com/ nature/debates/e-access/Articles/lawrence.html > Acesso em: 15 ago. 2008.

LEVACOV, M. Bibliotecas digitais: (r)evolução? Ciência da Informação, v. 26, n. 2, 1997.

MAIA, L. C. G.; CENDÓN, B. V. Uso de periódicos eletrônicos: um estudo sobre o Portal de PeriódicosCAPES na UFMG. In: ENCONTRO NACIONAL DA ASSOCIAÇÃO DE PESQUISA E PÓS-GRADUAÇÃO EM CIÊNCIA DA INFORMAÇÃO (ENANCIB), 6., 2005, Florianópolis. Anais eletrônicos... Florianópolis : UFSC, 2005.

MARCONDES, C. H.; SAYÃO, L. F. Documentos digitais e novas formas de cooperação entre sistemas de informação em C\&T. Ciência da Infomação, v. 31, n. 3, p. 42-54, 2002.

MARTÍNEZ-SILVEIRA, M. S.; ODDONE, N E. Information-seeking behavior of medical residents in clinical practice. In: CONGRESSO MUNDIAL DE INFORMAÇÃO EM SAÚDE E BIBLIOTECAS, 9.,2005, Salvador. Trabalhos apresentados... Disponível em: <http://www.icml9.org/program/track10/public/documents/ Martha\%20Silvia\%20Martinez-Silveira-152353.doc>. Acesso em 24 abr. 2006.

MARTINS, M. F. M. Uso do Portal CAPES na área biomédica: uma abordagem qualitativa. In: CONFERÊNCIA IBEROAMERICANA DE PUBLICAÇÕES ELETRÔNICAS NO CONTEXTO DA COMUNICAÇÃO CIENTÍFICA, 1., 2006, Brasília. Trabalhos apresentados... Brasília, 2006. Disponível em: $<$ http://portal.cid.unb.br/CIPECCbr/viewpaper.php?id=6>. Acesso em 24 abr. 2006.

MEYER, R. W. Monopoly power and electronic journals. Library Quarterly, n.67, n.4, p.325-349, 1997.

MONTEIRO, R. C. M. O grau de satisfação dos usuários do Portal de Periódicos da CAPES: estudo de caso na Universidade de Brasília e na Universidade Federal de Goiás. 2005. 131 f. Dissertação (Mestrado em Desenvolvimento Sustentável-Política e Gestão de Ciência e Tecnologia) - Centro de Desenvolvimento Sustentável, Universidade de Brasília, Brasília, 2005.

MORAVCSIK; Michael J.; MURUGESAN, Poovanalingam. Some results on the function and quality of citations. Social Studies of Science, v.5, n. 1, p. 86-92, 1975. 
MOREL, Regina Lúcia de Moraes; MOREL, Carlos Médicis. Um estudo sobre a produção científica brasileira, segundo os dados do Institute for Scientific Information (ISI). Ciência da Informação, v. 6, n. 2, p. 99-109, 1977.

NORONHA, Daisy Pires. Análise das citações das dissertações de mestrado e teses de doutorado em saúde pública (19901994): estudo exploratório. Ciência da Informação, v. 27, n. 1, p. 66-75, 1998. Disponível em: <www.ibict.br/cionline/>. Acesso em: 05 nov. 2008.

ODLYZKO, A. The economics of electronic journals. First Monday, v. 2, n. 8, 1997. Disponível em <http:/firstmonday.org/issues/ issue2 8/odlyzko/index.html>. Acesso em 10 jul. 2001.

PEEK, Robin. Electronic publishing goes up. Journal of the American Society for Information Science, v. 47, n. 9, p. 665-668, 1996.

PERITZ, B.C. On the Objectives of citation analysis: problems of theory and method. Journal of the American Society for Information Science., v. 43, n. 6, p. 448-451, 1992.

PINHEIRO, M. I. .S; SILVA, C. M.; DINIZ, E. Necessidade informacional via Internet: estudo do uso do Portal CAPES pelos docentes da UFMT - Campus de Rondonópolis. In: SEMINÁRIO NACIONAL DE BIBLIOTECAS UNIVERSITÁRIAS, 14., 2006, Salvador. Trabalhos apresentados... Disponível em: <http://www.snbu2006.ufba.br/soac/ viewabstract.php >. Acesso em 20 set. 2006.
PERIÓDICOS CAPES: o portal brasileiro de informações científicas. Disponível em: <http://www.periodicos.capes.gov.br/ portugues/index.jsp>. Acesso em 30 abr. 2008.

REIS, M. M. O. Acesso e uso do Portal de Periódicos CAPES pelos professores da Universidade Federal do Acre. 2005. 102 f. Dissertação (Mestrado em Ciência da Informação) - Universidade Federal de Santa Catarina, Florianópolis, 2005.

ROGERS S. Electronic journal usage at Ohio State University. College \& Research Libraries, v. 62, n. 1, p. 25-34, 2001.

ROGERS, S. HURT, C. S. How scholarly communication should work in the $21^{\text {st }}$ century. College and Research Libraries, v. 51, n. 1, p. 5-8, 1990 .

SCHAUDER, D. Electronic publishing of professional articles: attitudes of academics and implications for the scholarly communication industry. Journal of the American Society for Information Science, n. 45, p. 73-100, 1994.

VANZ, Samile Andréa de Souza; CAREGNATO, Sônia Elisa. Estudos de citação: uma ferramenta para entender a comunicação científica. Em Questão., v. 9, n. 2, p. 295-307, 2003.

WEINSTOCK, Melvin. Citation index. In: KENT, Allen; LANCOUR, Harold (Ed.). Encyclopedia of Library and Information Science. New York: M.Dekker, 1971.v. 5. p.19. 
